\title{
Variété et complexité des situations de travail et reconnaissance des acquis de métier: le travail des experts- évaluateurs
}

Otilia Holgado, Université de Sherbrooke, Canada,

Otilia.Holgado@usherbrooke.ca

Charles Allard-Martin, Université de Sherbrooke, Canada,

Charles.Allard-Martin@USherbrooke.ca 


\section{$\&$}

\section{REVUE HYBRIDE DE L'ÉDUCATION}

\section{Résumé}

Cet article examine le travail des professionnels expérimentés qui agissent à titre d'experts-évaluateurs dans le cadre du processus de reconnaissance des acquis de métier (RAM) au baccalauréat en enseignement professionnel (BEP) dans une université québécoise. Partant du constat que les concepts de « variété » et de « complexité » des situations de travail se situent au centre de l'activité évaluative, cet article tente de montrer comment ces concepts sont construits et mobilisés par les experts-évaluateurs dans l'analyse des dossiers des étudiants candidats à la RAM. Pour finir, l'article propose quelques pistes pour la formation des experts-évaluateurs, dans une visée d'homogénéisation des pratiques évaluatives.

Mots-clés: reconnaissance des acquis de métier; compétence professionnelle; évaluation; formation professionnelle; situation de travail 


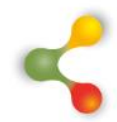

\section{REVUE HYBRIDE DE L'ÉDUCATION}

\section{Problématique}

\section{La reconnaissance des acquis de métier : une démarche obligatoire}

Offert dans plusieurs universités québécoises, le baccalauréat en enseignement professionnel (BEP) est un diplôme de formation à l'enseignement de 120 crédits ouvert aux travailleurs expérimentés des métiers qualifiés (coiffure, mécanique, etc.) qui débutent ou qui souhaitent débuter une carrière en enseignement dans un centre de formation professionnelle (enseignant de coiffure, enseignant de mécanique, etc.). Ces 120 crédits se divisent en deux blocs : un premier bloc de 90 crédits conduit à la licence d'enseignement; un deuxième bloc de 30 crédits mène à l'obtention du BEP et du brevet d'enseignement (Aubé et al., 2009). Le baccalauréat en enseignement professionnel se distingue des autres programmes de formation universitaire à l'enseignement par le fait qu'il met l'accent sur une formation en enseignement adaptée à la transition de la pratique d'un métier qualifié de l'industrie ou des services vers l'enseignement de ce même métier (Balleux, 2003). Dans ce sens, l'une des spécificités de cette formation est la possibilité de «se faire reconnaître jusqu'à 27 crédits, réservés à la reconnaissance de l'expérience de travail en lien avec la discipline professionnelle à enseigner " (Zourhlal et Coulombe, 2014, p. 169).

À travers le processus de reconnaissance des acquis de métier (RAM), les étudiants rédigent un portfolio de compétences liées au métier qu'ils ont déjà pratiqué et qu'ils commencent à enseigner. Ils ciblent des compétences du référentiel de compétences du programme menant au diplôme d'études professionnelles (DEP) qui prépare au métier présenté et mettent en valeur, par écrit, leur niveau d'expertise pour chacune des compétences sélectionnées. Le niveau d'expertise des compétences des étudiants est déterminé par la complexité et la variété des réalisations professionnelles décrites dans leur portfolio. Pour chaque compétence choisie, l'autoévaluation du niveau de complexité et de variété des situations de travail vécues permet aux étudiants de déterminer le nombre de crédits à demander en reconnaissance des acquis de métier, soit entre un et quatre crédits. Se considérant comme des professionnels expérimentés, la plupart des étudiants demandent 3 crédits par compétence, l'attribution de 4 crédits étant réservée à des réalisations d'exception, comme des innovations reconnues pour avoir fait évoluer considérablement les pratiques de son métier (brevets, conception de produits commercialisés à grande échelle, etc.).

\section{Le processus de reconnaissance des acquis de métier et ses particularités}

La finalité du processus de RAM est de traduire en crédits universitaires l'expérience professionnelle acquise par les étudiants avant leurs débuts dans l'enseignement. Pour y arriver, l'évaluation des dossiers 


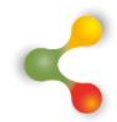

\section{REVUE HYBRIDE DE L'ÉDUCATION}

de RAM est confiée à un comité d'évaluation qui a comme responsabilité de rendre un verdict sans appel quant au nombre de crédits alloué par dossier. Le comité, présidé par un représentant du BEP, est composé d'au moins trois personnes. Pour chaque dossier, un expert du métier concerné assure le rôle d'évaluateur principal. Dans ce texte, nous allons l'appeler « expert-évaluateur ». Il est considéré comme évaluateur externe, car il ne participe pas à la formation des étudiants du BEP et ne connait pas le candidat. Lors d'une réunion de comité, un expert-évaluateur peut évaluer entre un et trois dossiers.

Sur un comité de RAM siègent plusieurs experts, de métiers différents, ayant chacun la responsabilité d'au moins un dossier. Pour chaque dossier, l'expert-évaluateur fait part de son évaluation aux autres membres du comité en présentant les points forts et les faiblesses du candidat en termes de compétence professionnelle. À la suite des discussions qui en découlent, le comité rend son verdict final. Les expertsévaluateurs sont sélectionnés par les responsables de la RAM de chaque université. D'une université à l'autre, les critères de sélection varient, mais partagent plusieurs similarités, telles une expérience de travail solide des experts, la connaissance du programme d'études visé par la demande ainsi que le fait d'être eux-mêmes des enseignants des centres de formation professionnelle et donc, la plupart du temps, titulaires d'un BEP pour le métier visé, ce qui garantit leur connaissance du processus de RAM (l'ayant déjà traversé eux-mêmes par le passé).

Comme l'explique Werquin (2010), une des particularités importantes à prendre en compte pour l'évaluation dans le processus de reconnaissance d'acquis non formels (ou de métier dans le cas de cette recherche) est le fait que l'on " déconnecte complètement, dans le temps et dans l'espace, la relation entre le processus d'apprentissage et l'utilisation de ces apprentissages. II n'y a plus aucune relation entre l'enseignement et l'évaluation » (p.17). Cette dimension est renforcée par le fait que l'évaluation des dossiers de RAM est effectuée par des expertsévaluateurs qui ne participent pas directement à la formation des étudiants concernés par la demande.

\section{L'instrumentation des experts-évaluateurs}

Les experts-évaluateurs du processus de RAM au BEP assurent, au quotidien, plusieurs rôles:

1. Professionnels expérimentés dans leurs métiers respectifs, qu'ils ont exercés pendant longtemps et qu'ils continuent, pour certains, à exercer à temps partiel ;

2. Enseignants en centre de formation professionnelle ;

3. Experts-évaluateurs de dossiers de RAM pour le BEP, rôle qu'ils assurent ponctuellement. 


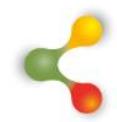

\section{REVUE HYBRIDE DE L'ÉDUCATION}

Cette multitude de rôles influence le jugement professionnel et évaluatif que les experts portent sur le contenu à évaluer. Les compétences en évaluation, ils les ont tout d'abord développées par le biais de leur rôle d'enseignant en centre de formation professionnelle. Or, l'évaluation en situation d'enseignement et le mandat d'évaluateur RAM au BEP divergent au niveau de l'instrumentation. En centre de formation professionnelle, les enseignants recourent «à des instruments d'observation adaptés à l'objectif poursuivi » (gouvernement du Québec, 2003, p. 16). Pour la RAM au BEP, ils évaluent des faits relatés par écrit par les candidats. L'observation directe est impossible tout comme l'explication orale, car le candidat et le jury ne se rencontrent pas et ne communiquent pas pour préserver l'anonymat des experts-évaluateurs. De plus, comme mentionné précédemment, les experts-évaluateurs sont des personnes externes, qui ne participent pas à la formation des étudiants du BEP.

Cette réalité amène un double travail d'analyse et d'interprétation pour les évaluateurs :

1. Analyse et interprétation des directives et des critères d'évaluation tels que présentés dans l'outil d'évaluation mis à disposition par la personne responsable de la RAM au BEP ;

2. Analyse et interprétation du contenu du dossier de RAM à évaluer.

Romainville (2011) explique que pour objectiver la subjectivité inhérente au processus d'évaluation, il faut légitimer le point de vue spécifique des évaluateurs, mais aussi «que ce point de vue ait été l'objet d'une explicitation préalable auprès des étudiants, de sorte que l'évaluation ne s'apparente pas au "jeu du chat et de la souris" » (p.6). Cette explicitation préalable entre l'expert-évaluateur et l'étudiant ne se fait pas directement, elle est déléguée à un tiers, le chargé de cours qui accompagne les étudiants dans la rédaction du dossier de RAM. Sans lien direct avec les candidats, les experts-évaluateurs devraient se doter d'instruments objectifs pour effectuer leur mandat (Mayeux et Mayen, 2009). Comme le mentionnent Mayen et Tourmen (2009) en reprenant l'idée de Dewey, "une expérience est vécue dans la continuité d'expériences antérieures » qui «préfigurent très largement la manière dont l'expérience actuelle est appréhendée» (p. 60). Toutefois, si l'expérience de métier des experts-évaluateurs est indispensable à l'évaluation, celle-ci amène également une certaine subjectivité. Cortessis (2010) montre que le jugement global et subjectif des évaluateurs dans un processus de reconnaissance des acquis et de compétences est inévitable, et ce, malgré l'instauration d'outils d'évaluation. Effectivement, une recherche exploratoire nous a permis de montrer que certains expertsévaluateurs ont tendance à déconsidérer les dossiers de RAM quand les techniques de métier décrites ne correspondaient pas à leurs techniques de prédilection (Holgado, 2017). Ainsi, la question qui se pose est celle de la formation et de l'encadrement de l'activité des experts-évaluateurs afin d'assurer une évaluation juste et équitable pour les candidats. Dans le 


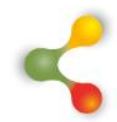

\section{REVUE HYBRIDE DE L'ÉDUCATION}

contexte analysé, les experts-évaluateurs suivent une formation préalable et reçoivent des consignes d'évaluation, mais à quel point ces ressources arrivent-elles à baliser l'utilisation réelle qu'ils feront de leur propre expérience évaluative acquise en tant que professionnels et d'enseignants?

La recherche que nous présentons examine l'activité évaluative des experts-évaluateurs en RAM, activité située à la croisée de trois aspects : le dossier du candidat à évaluer, les consignes reçues et leur propre expérience mobilisée au service de l'évaluation. Plus spécifiquement, nous nous intéressons à la construction faite par les experts-évaluateurs des deux notions centrales de l'évaluation en RAM : la complexité et la variété des situations de travail décrites par les candidats dans leurs dossiers.

\section{Cadre théorique ou conceptuel}

\section{Contourner la notion de compétence pour mieux l'évaluer}

La notion de compétence est polysémique (Jonnaert, 2011) et sa signification dans le cadre de la RAM au BEP est bivalente. D'une part, cette notion renvoie aux référentiels de compétences disponibles pour chacun des programmes d'études professionnelles et sur lesquels les étudiants s'appuient pour identifier les compétences qu'ils ont la possibilité de se faire reconnaître. D'autre part, dans le portfolio qu'ils rédigent, les candidats à la RAM tentent de mettre en évidence les compétences-enacte, c'est-à-dire les compétences du référentiel, mais qui ont été construites, enrichies, structurées et marquées par des expériences personnelles vécues sur le marché du travail. Emprunté au concept de connaissance-en-acte (Vergnaud, 1990), le suffixe « en-acte » signifie que les compétences sont « entièrement intégrées dans l'exécution de l'activité et échappent, de ce fait, à la conscience et à leur explicitation par le sujet » (Coulet, 2011, p. 14). Pour le processus de RAM, il s'agit de compétences construites par les candidats sur une durée longue, à travers des expériences de travail nombreuses, mais hétérogènes, difficiles à capitaliser de manière structurée. Mayen, Métral et Tourmen, qui se sont intéressés à l'activité des jurys en validation des acquis d'expérience, définissent la compétence comme étant «la relation dynamique d'une personne avec des situations ou des classes de situations » (Mayen, Métral et Tourmen, 2010, p. 32).

Toutefois, définir la compétence ne suffit pas pour pouvoir l'identifier et la reconnaître. Dans une recherche sur la VAE, Savoyant et Mayen (2009) ont rappelé que dans le processus de validation de ses compétences, un candidat ne s'engage pas seulement avec ses compétences formelles, mais avec «toute sa personne». Réduire l'expérience de toute une vie professionnelle à un texte relativement court peut être éprouvant pour les candidats, car il s'agit de la synthétiser sans 


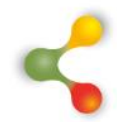

\section{REVUE HYBRIDE DE L'ÉDUCATION}

l'appauvrir. Or, la littérature a montré la difficulté des experts-évaluateurs à s'inscrire dans une démarche d'évaluation qui demande de "s'affranchir d'un mode évaluatif dont ils ont la maîtrise, pour s'engager dans un nouveau mode à construire. Ce passage doit s'opérer en faisant le deuil d'une évaluation des connaissances, interdite par la loi si elle est déconnectée de l'expérience professionnelle » (Cuvillier et Faudière, 2009). Dans ces conditions, les experts-évaluateurs doivent être formés pour faire l'analyse adéquate.

En RAM, la notion de compétence se retrouve également reliée à un aspect plus intime : qu'est-ce qu'un professionnel compétent? Le Boterf explique qu'«être compétent c'est non seulement disposer de compétences et les mobiliser, mais c'est aussi mettre en œuvre un déroulé de choix, de décisions et d'actions réellement mis en œuvre par une personne pour faire face aux exigences prescrites d'une situation " (Le Boterf, 2017, p. 4). II est ainsi possible qu'« une personne pourrait avoir beaucoup de compétences et ne pas être compétente » (Le Boterf, 2000, p. 11). De son côté, Legendre (2001) considère qu'être compétent est la capacité d'un individu à choisir et à appliquer des ressources internes et externes pour agir efficacement dans une situation spécifique dans un contexte donné. Enfin, Coiduras et Carrera (2010) abordent la notion de personne compétente en expliquant que «la personne qui agit de façon compétente a développé une aptitude mentale pour saisir et donner du sens aux événements, aux phénomènes, et construire des hypothèses pertinentes afin de gérer la variété des tâches » (p. 99). Pour la didactique professionnelle, être compétent revient à maîtriser les situations telles qu'elles se présentent au travailleur, c'est-à-dire d'une part avec leur degré de complexité et d'autre part avec leur degré de familiarité, de "déjà-vu " ou le contraire, de nouveauté, de singularité, ce qui fait la variabilité des situations. Au travail, être compétent demande d'agir avec les situations et parfois même d'agir sur les situations, les modifier pour les rendre maîtrisables. Ainsi, un mécanicien compétent qui n'arrive pas, avec les outils et les procédures habituels, à accéder à un boulon permettant d'extraire une pièce mécanique d'un équipement sans enlever préalablement d'autres pièces, ce qui ralentirait l'intervention, va tenter d'envisager un autre angle d'attaque, par exemple utiliser d'autres outils qu'il a à sa disposition et qui ne sont pas nécessairement conçus pour réaliser cette tâche, ou encore - tel qu'un candidat le montre dans son portfolio - se créer soi-même un accès en faisant un petit trou sur le côté du boitier de protection de l'équipement, trou qu'il prendra le soin de colmater par la suite.

Les différentes définitions de la compétence et du professionnel compétent montrent que la compétence en soi n'est pas directement observable. Hébrard précise que «seules les activités le sont [observables] et c'est sur la base de l'observation et de l'analyse de celles-ci que l'on pourra inférer l'existence d'une compétence » (Hébrard 2013, p. 19-20). La compétence est cependant déductible à travers les expériences de travail 


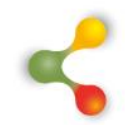

\section{REVUE HYBRIDE DE L'ÉDUCATION}

décrites par les individus. C'est pour cela que la notion d'expérienceévénement, telle que décrite par Mayen (2009), s'avère importante. Mayen explique que «le retour sur l'expérience, quels qu'en soient les objectifs, se fonde sur l'expérience comme événement parce que seuls les événements ont des propriétés qui rendent l'analyse possible » (Mayen, 2009, p. 768). Les événements décrits dans les dossiers de RAM sont la résultante d'un retour réflexif de l'étudiant sur sa propre expérience professionnelle.

Nous retenons donc les aspects suivants:

1. le choix des compétences à valider est limité et cadré par un référentiel ;

2. la compétence professionnelle est «en-acte» donc difficile à décrire, à identifier et à quantifier, mais possible à analyser indirectement, à travers les expériences décrites par les candidats. Un travail d'analyse et de corrélation est cependant nécessaire ;

3. les situations occupent une place centrale dans l'évaluation de la compétence.

Par conséquent, le travail des experts-évaluateurs en RAM se résume ainsi : à la lecture des portfolios préparés par les candidats à la RAM, les experts-évaluateurs sont invités à prendre connaissance des expériences de travail vécues par les candidats et de les calquer sur les compétences clés du métier visé. Ils doivent chercher à identifier des éléments leur permettant de reconstituer les situations vécues par les candidats pour comprendre les difficultés qui se sont présentées à eux et ce qui les a déterminés à agir d'une manière plutôt que d'une autre. Enfin, ils vont estimer le niveau de compétence et attribuer une valeur quantifiable en crédits universitaires.

\section{Analyser le travail des experts-évaluateurs}

Permettant de mettre à jour « les compétences que chacune des tâches accomplies convoque ainsi que la part d'explicitation et d'initiative à multiplier dans une situation où tout n'est pas dit ou écrit » (Clauzard, 2008, p.4), l'analyse du travail est au fondement même de la didactique professionnelle. Elle met en relation trois éléments : la tâche, la situation et l'activité (figure 1). 


\section{REVUE HYBRIDE DE L'ÉDUCATION}

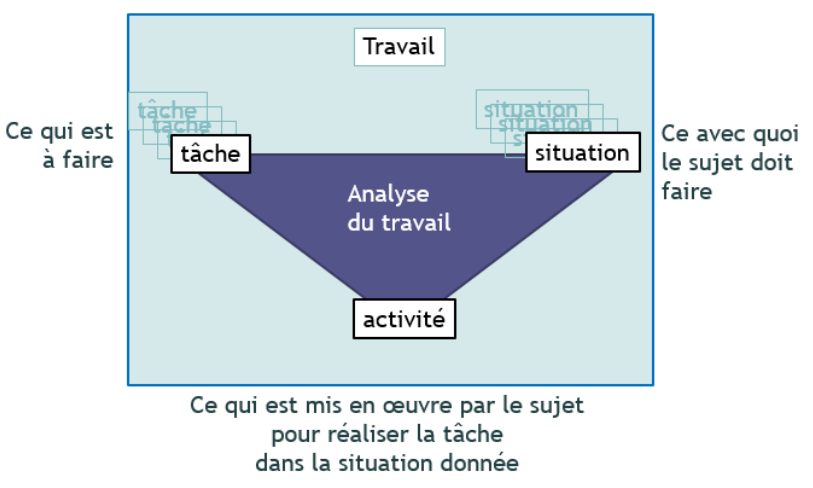

Figure 1 : Comment analyser le travail pour la formation professionnelle? (Holgado, 2015)

La tâche correspond à ce qui est à faire (ou ce qui est demandé). La situation regroupe l'ensemble des conditions qui déterminent la réalisation de la tâche : le contexte, l'environnement proche, les ressources humaines (aide) et matérielles (outils, matière première) disponibles. En prenant en compte ces éléments et leur variabilité, toute situation de travail est unique (Mayen, Métral et Tourmen, 2010). L'analyse du travail permet de dégager des éléments qui montrent que les situations sont plus ou moins complexes: les buts à atteindre, le degré de variabilité, les résultats attendus, le conflit entre les résultats productifs et la protection de soi, etc. (Mayen, Métral et Tourmen, 2010). Enfin, l'activité représente l'ensemble d'actions (gestes, choix, réflexions, prises de conscience, hésitations, décisions, erreurs, etc.) mises en œuvre pour réaliser une tâche donnée dans une situation donnée. Le caractère personnel de l'activité permet aux professionnels d'agir différemment dans des situations semblables et obtenir des résultats comparables. En effet, l'activité dépend de la manière dont chacun se représente la tâche et analyse la situation pour repérer les ressources disponibles.

Pour ce qui est du travail des experts dans le cadre de la validation des acquis de l'expérience (VAE), Mayen et Métral (2008) proposent d'explorer quatre dimensions :

- Les situations que [le candidat] a vécues et leur enchaînement ;

- La manière d'agir du candidat et sa conformité au regard de celle attendue de la part d'un professionnel expérimenté ;

- Les ressources (connaissances, outils, méthodes) utilisées par le candidat dans ses raisonnements dans et sur l'activité ;

- Les contraintes inhérentes aux situations rencontrées par le candidat et la manière dont elles ont pu constituer un frein [ou un levier] au développement des compétences (p. 190). 


\section{6}

\section{REVUE HYBRIDE DE L'ÉDUCATION}

Ces quatre dimensions viennent objectiver l'activité évaluative dans le processus de RAM au BEP. Cependant, le travail des expertsévaluateurs est aussi teinté de plusieurs éléments de subjectivité, notamment en raison de leur jugement professionnel et évaluatif. Tel que décrit par Allal et Lafortune (2008), le jugement professionnel est un processus de prise de décisions rigoureux, cohérent et transparent qui suppose «la collecte d'informations à l'aide de différents moyens, la justification du choix des moyens en lien avec les visées ou intentions et le partage des résultats de la démarche dans une perspective de régulation " (p. 4). Le jugement évaluatif demande le développement et l'application de " procédures d'évaluation rigoureuses, transparentes, justes et équitables pour l'ensemble des étudiants, tout en s'assurant de leur adaptation aux situations singulières qui l'exigent »(Chaumont et Leroux, 2018, p. 28). Le jugement évaluatif des experts-évaluateurs n'est cependant pas le même que celui d'un enseignant traditionnel. Mayen et Métral (2009) ont étudié la construction du jugement en VAE et en ont démontré la complexité, en expliquant que ce jugement est influencé autant par des éléments micros (le dossier à évaluer, la séance d'échange avec le jury, le vécu et les valeurs de l'évaluateur, etc.) que par des éléments macro (le monde de la formation, le contexte sociopolitique, etc.). En considérant cette complexité et en reprenant l'idée que les experts-évaluateurs assurent différents rôles, ils peuvent être tentés de se référer à leur expérience, mais aussi à des aspects théoriques et techniques qu'ils enseignent au DEP pour juger si les situations de travail décrites dans le portfolio prouvent que le candidat a effectivement atteint le niveau de compétence correspondant au nombre de crédits demandés. Ainsi, la place que prennent l'expérience professionnelle, la formation antérieure et l'identité des experts-évaluateurs ne peut pas être négligée, mais, comme le souligne Savoie-Zajc (2013), la rigueur, la transparence et l'équité sont nécessaires pour contrôler l'influence que peuvent avoir les caractéristiques personnelles de l'évaluateur sur le travail d'évaluation.

\section{Méthodologie}

La présente recherche qualitative de type exploratoire s'inscrit dans une démarche globale qui vise à améliorer la formation des expertsévaluateurs recrutés pour la RAM au BEP. Les données utilisées ici correspondent au déroulement de plusieurs comités d'évaluation pour la RAM. Durant ces rencontres de comités d'environ une heure chacune, les experts-évaluateurs présentent leurs conclusions et échangent au sujet de chaque dossier. La durée des échanges varie selon le nombre de commentaires que chacun des membres a à transmettre en ce qui a trait à la qualité et la pertinence du dossier évalué. Les membres du comité peuvent demander à l'expert-évaluateur responsable d'un dossier de préciser certains aspects de son évaluation pour s'assurer que le nombre de crédits accordé est juste et équitable. Les échanges concernant quatorze dossiers ont été transcrits en verbatims pour l'analyse dans le but 


\section{8}

\section{REVUE HYBRIDE DE L'ÉDUCATION}

de cerner les décalages qui existent entre : la définition de la variété et de la complexité telle qu'elle ressort de l'outil d'évaluation mis à disposition, l'interprétation que font les experts-évaluateurs de ces mêmes critères et l'application des critères à l'analyse des dossiers des étudiants. Les portfolios évalués concernaient neuf secteurs de métier différents: assistance dentaire (1), plomberie-chauffage (2), briquetage-maçonnerie (1), esthétique (1), intervention en sécurité incendie (2), mécanique de véhicules lourds (1), charpenterie-menuiserie (2), transport par camion (2), ainsi que santé, assistance et soins infirmiers (2).

L'analyse des données a été effectuée en suivant la méthode d'analyse inductive générale, telle que décrite par Blais et Martineau (2006). Cette méthode «s'appuie sur différentes stratégies utilisant prioritairement la lecture détaillée des données brutes pour faire émerger des catégories à partir des interprétations du chercheur qui s'appuient sur ces données brutes » (Blais et Martineau, 2006, p. 3). À la suite de l'analyse des quatorze verbatims, huit catégories conceptuelles ont été créées pour le critère de variété et onze pour le critère de complexité. Chaque catégorie représente un élément sur lequel un ou plusieurs experts se basent pour définir chacun desdits critères dans leur activité évaluative. Ces éléments ont ensuite été confrontés avec les critères qui émergent de l'outil d'évaluation mis à disposition pour encadrer le processus d'évaluation.

\section{Résultats}

Parmi les échanges portant sur les quatorze dossiers analysés, sept mettaient explicitement de l'avant la notion de variété et douze mettaient explicitement de l'avant la notion de complexité. Le tableau 1 présente la comparaison entre l'outil d'évaluation mis à disposition et la construction par les experts-évaluateurs de la notion de variété des situations, tandis que le tableau 2 présente la même comparaison pour la notion de complexité. Les éléments présents dans la colonne "Construction des critères d'évaluation par les experts-évaluateurs " correspondent aux catégories conceptuelles qui ont été créées à partir des échanges qui ont eu lieu au sein des comités d'évaluation, donc entre les expertsévaluateurs et les autres membres du comité. Les tableaux présentent aussi le nombre d'échanges qui traitaient explicitement de chaque catégorie conceptuelle identifiée. 


\section{REVUE HYBRIDE DE L'ÉDUCATION}

Tableau 1 : Définition de la notion de variété des situations selon l'outild'évaluation du processus de RAM au BEP et selon les experts-évaluateurs

\begin{tabular}{|c|c|}
\hline $\begin{array}{llr}\text { Selon Extrait } & \text { de } & \text { l'outil } \\
\text { d'évaluation } & \text { mis } & \text { à } \\
\text { disposition } & & \\
\end{array}$ & 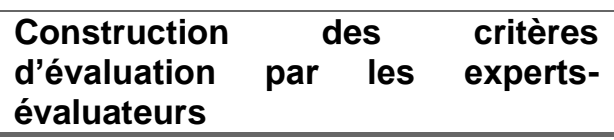 \\
\hline $\begin{array}{l}\text { "Au moins } 2 \text { exemples sont } \\
\text { suffisamment différents } \\
\text { pour prouver la variété des } \\
\text { situations : tâches } \\
\text { différentes, contextes } \\
\text { différents, modes d'action } \\
\text { différents et types de } \\
\text { résultats différents. " }\end{array}$ & $\begin{array}{l}\text { Variété des tâches effectuées }(7 / 7) \\
\text { Variété des contextes/domaines de } \\
\text { travail }(5 / 7) \\
\text { Variété des rôles exercés }(1 / 7) \\
\text { Variété des clientèles }(1 / 7) \\
\text { Variété des équipements utilisés }(1 / 7) \\
\text { Voir la personne dans l'action }(1 / 7) \\
\text { Présenter des exemples complets } \\
\text { (1/7) } \\
\text { Aborder tous les aspects d'une } \\
\quad \text { compétence }(1 / 7)\end{array}$ \\
\hline
\end{tabular}

Tableau 2: Définition de la notion de complexité selon l'outil d'évaluation du processus de RAM au BEP et selon les experts-évaluateurs

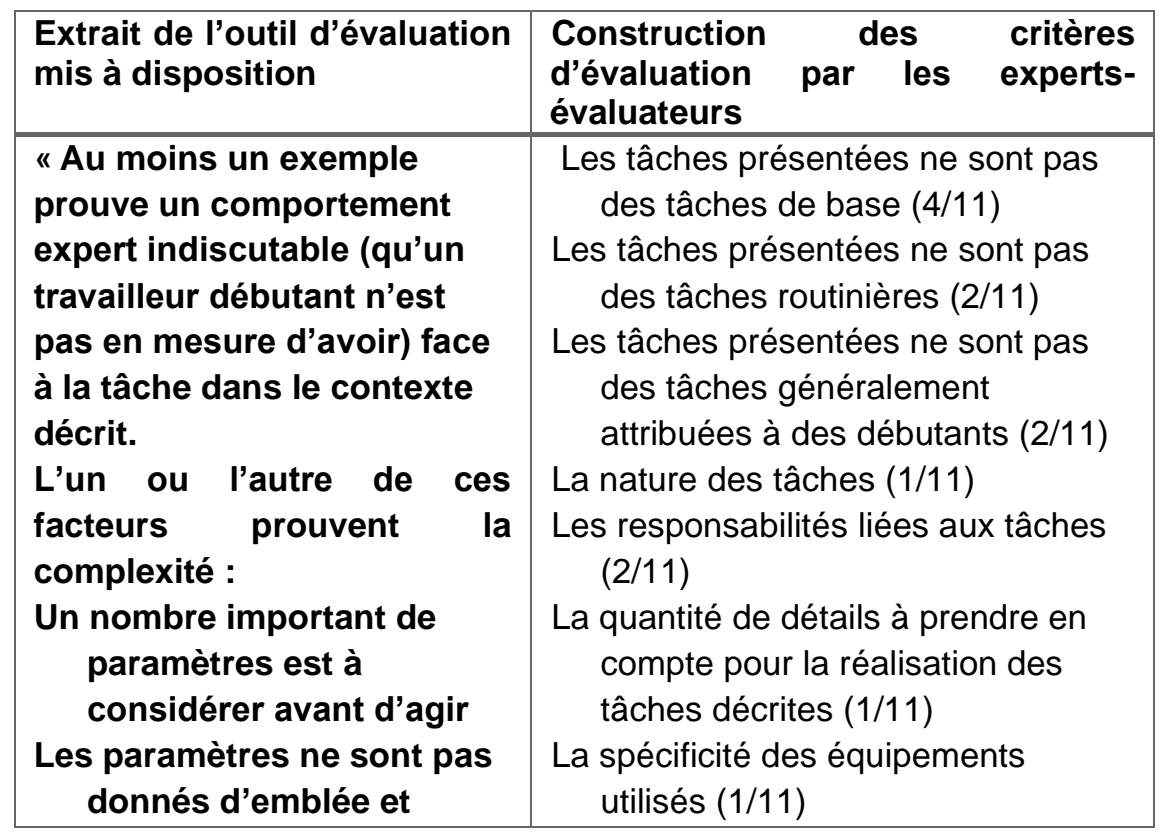




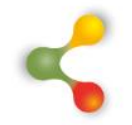

REVUE HYBRIDE DE L'ÉDUCATION

\begin{tabular}{|c|c|}
\hline $\begin{array}{l}\text { doivent être identifiés par } \\
\text { le travailleur } \\
\text { La procédure habituelle ne } \\
\text { peut s'appliquer dans le } \\
\text { contexte donné (la raison } \\
\text { est justifiée) et une } \\
\text { nouvelle procédure est à } \\
\text { concevoir sur place } \\
\text { La réalisation de la tâche } \\
\text { nécessite la mise en } \\
\text { application de } \\
\text { connaissances multiples, } \\
\text { dont certaines acquises } \\
\text { par la formation et } \\
\text { d'autres par } \\
\text { l'expérience. » }\end{array}$ & $\begin{array}{l}\text { Absence d'erreur dans les techniques } \\
\text { décrites ( } 1 / 11) \\
\text { Le contenu décrit provient de la } \\
\text { pratique du métier par la personne } \\
\text { (pas de description théorique) } \\
\text { ( } 1 / 11) \\
\text { Le degré de généralité (pas de } \\
\text { description trop générale) }(3 / 11) \\
\text { La personne est engagée dans } \\
\text { l'action (emploi des verbes } \\
\text { d'action à la } 1 \text { re personne du } \\
\text { singulier) }(2 / 11) \\
\text { Le contenu est suffisant (quantité de } \\
\text { texte) }(5 / 11)\end{array}$ \\
\hline
\end{tabular}

\section{Discussion}

Plusieurs constats peuvent être formulés suite à la comparaison entre la définition des notions de variété et de complexité des situations de travail donnée par l'outil d'évaluation et celle que se construisent les experts-évaluateurs de ces mêmes notions.

\section{L'outil d'évaluation mis à disposition est partiellement utilisé par les experts-évaluateurs}

Le premier constat que nous pouvons faire est que tous les éléments présents dans l'outil d'évaluation mis à disposition ne sont pas repris par les experts-évaluateurs dans la construction des critères de variété et de complexité des situations. En revanche, les experts introduisent des indicateurs auxquels l'outil d'évaluation ne fait pas référence.

Pour le critère de variété, se sont la variété des tâches (7/7) et celle des contextes de réalisation de ces tâches (5/7) qui se retrouvent à la fois dans l'outil d'évaluation et chez les experts-évaluateurs. Se sont, indiscutablement, les deux indicateurs les plus pertinents pour les expertsévaluateurs. Toutefois, la variété des contextes est associée par les experts-évaluateurs à la diversité des environnements professionnels dans lesquels le candidat a évolué ; cet indicateur pénalise les expériences vécues dans un contexte de mobilité plus restreint. Ainsi, un candidat qui a travaillé dans la construction, aussi bien dans le secteur résidentiel, que commercial et industriel, sera considéré plus facilement compétent par les experts-évaluateurs qu'un autre candidat qui a vécu la plupart de ses 


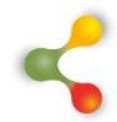

\section{REVUE HYBRIDE DE L'ÉDUCATION}

expériences de travail dans le secteur résidentiel uniquement, même si ce dernier a pu développer, par la récurrence des tâches, des compétences plus solides dans le secteur concerné.

Quant aux indicateurs de variété issus de l'outil d'évaluation et qui ne sont pas repris par les experts-évaluateurs, nous retenons la variété des modes d'action et la variété des résultats. Selon la didactique professionnelle, ces deux aspects sont essentiels pour mettre en évidence la compétence des travailleurs. La variété des modes d'action est mobilisée par Vergnaud (2001) pour définir la compétence d'un travailleur: si une personne est capable d'utiliser plusieurs procédures pour la même tâche, elle prouve sa capacité à s'adapter plus aisément aux différentes situations qui peuvent se présenter à elle. Sa compétence est rattachée ici à sa capacité à répondre à des considérations économiques et sociales diverses : choisir la procédure la plus rapide, la moins dispendieuse et la plus sécuritaire dans une situation donnée. La variété des formes que peuvent prendre les résultats réfère au fait que le résultat obtenu s'ajuste à la demande ou, selon le cas, aux besoins des clients et témoigne ainsi d'un souci fonctionnel de la part du travailleur, qui est également une forme de compétence.

Les experts-évaluateurs construisent trois indicateurs de variété qui ne sont pas prévus par l'outil d'évaluation : la variété des rôles, la variété des clientèles desservies et la variété d'équipements utilisés. La variété des rôles introduit un aspect intéressant, celui du regard posé sur le métier. Ainsi, un travailleur devenu contremaître ou chef de chantier, est considéré avoir vécu une palette plus large d'expériences professionnelles et obtiendra plus facilement le qualificatif d'expert (et un nombre de crédits plus élevé) qu'un candidat qui a eu une évolution hiérarchique plus limitée. En revanche, les experts-évaluateurs sont très exigeants dans l'analyse des expériences décrites par un candidat qui est arrivé dans le métier pour assurer directement une fonction d'encadrement.

Pour le critère de complexité, les consignes fournies aux expertsévaluateurs à travers l'outil d'évaluation semblent difficiles à traduire en indicateurs, car les experts-évaluateurs n'en tiennent pas vraiment compte. Les indicateurs construits par les experts-évaluateurs sont concrets, mais simplifiés; ils portent davantage sur des caractéristiques des tâches réalisées par les candidats que sur les situations de réalisation de ces tâches. En effet, les deux premiers indicateurs construits par les expertsévaluateurs (tableau 2) sont le type de tâches (communes, routinières, critiques, rares, etc.) et leur nature (tâche de production, de réparation ou de remise en état, de diagnostic, de conception, etc.). Plus encore, les experts classifient les tâches en les associant à une reconnaissance socioprofessionnelle, construite sur la base de l'affirmation suivante : au travail, les tâches sont attribuées à celui qui est capable de les réaliser. Ainsi, l'ancienneté et le statut du travailleur deviennent, pour les expertsévaluateurs, des indicateurs de compétence, tout comme le niveau de 


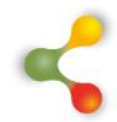

\section{REVUE HYBRIDE DE L'ÉDUCATION}

responsabilité assuré au travail. Dans ce sens, les experts-évaluateurs s'appuient sur l'idée a priori selon laquelle l'exécution des tâches par les apprentis débutants est toujours assistée et encadrée par un collègue plus expérimenté ou par un contremaître. Les experts expriment des doutes quand un candidat affirme avoir eu, en tant qu'apprenti débutant, la responsabilité d'une tâche ou d'une série de tâches, même parmi les plus simples, mais s'attendent à ce que les apprentis plus anciens bénéficient de plus d'autonomie. La prise de décision et le travail autonome, les experts le reconnaissent uniquement aux contremaîtres et aux travailleurs indépendants.

Un autre indicateur de complexité pour les experts-évaluateurs réfère aux équipements utilisés par les candidats. Les experts évaluent la maîtrise des équipements et des instruments de travail en lien avec leur fiabilité : un outil réputé fragile, capricieux ou difficile à manipuler apportera une plus grande valeur ajoutée à la compétence du candidat.

Enfin, les techniques et les procédures employées dans la réalisation des tâches sont également considérées comme des indicateurs de complexité. Les experts-évaluateurs, ayant déjà construit par expérience un registre de "bonnes » manières de faire et d'agir, recherchent chez le candidat la conformité des manières de faire avec la situation telle qu'ils se la représentent.

\section{Variété et complexité des situations, deux critères inégaux}

Comparativement au critère de variété, le critère de complexité semble être moins accessible aux experts-évaluateurs, qui l'utilisent comme un critère « fourre-tout ». Il est difficile de dégager une interprétation commune de ce critère pour les experts-évaluateurs. Pour certains, la complexité est liée au type et à l'étendue des tâches réalisées. Pour d'autres, c'est l'activité complexe qui est un indicateur pertinent ; ceux-ci vont chercher à évaluer les manières de faire et d'agir des travailleurs et montreront un intérêt marqué pour la singularité de l'activité. D'autres encore rattachent la complexité à des situations hors du commun, soit inhabituelles, soit d'une grande difficulté ou présentant un risque élevé. Pourtant, selon la didactique professionnelle, la situation se trouve de manière invariable au cœur de la démonstration de la compétence professionnelle. Qu'elle soit réduite à quelques paramètres ou nécessitant une prise d'information et une conceptualisation prolongée, qu'elle soit facile ou difficile à cerner, la situation représente la part d'environnement qui impacte directement et inévitablement l'activité du travailleur.

Si le critère de complexité ne fait pas consensus, les expertsévaluateurs s'accordent en revanche sur celui de variété, qu'ils associent à «suffisamment différent»: la compétence est prouvée lorsque le candidat a réalisé des tâches suffisamment différentes, dans des contextes de travail suffisamment différents, en assurant des rôles suffisamment 


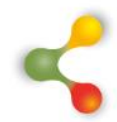

\section{REVUE HYBRIDE DE L'ÉDUCATION}

différents, etc. Il est cependant difficile d'identifier avec précision ce que les experts entendent par suffisamment différent.

En conclusion, à la lumière des constats que nous venons de présenter, la construction par les experts-évaluateurs des critères de complexité et variété reste très individuelle et insuffisamment centrée sur les situations vécues par les candidats. Les experts préfèrent appuyer leur évaluation sur des aspects qui leur sont plus familiers et qui sont plus faciles à interpréter, comme les tâches réalisées ou les rôles assumés par les candidats.

\section{Perspectives}

Plusieurs travaux portant sur le rôle des experts-évaluateurs dans le processus d'évaluation en RAM (ou en VAE en Europe) ont montré que les experts-évaluateurs ne se réfèrent pas directement et strictement au référentiel des compétences tel que fourni (Cunha et Santos, 2019) et n'emploient pas les outils d'évaluation tels que mis à disposition, préférant construire leurs propres références et outils à partir de trois types de ressources :

- Ressources mises à disposition pour évaluer: consignes, formation, critères et indicateurs, etc.

- Leur propre expérience de travail,

- leur propre expérience d'évaluation, antérieure (capitalisée) et simultanée (comparaison entre dossiers) dans la recherche d'une équité (Mayeux et Mayen, 2009).

Notre recherche a permis de ressortir, explicitement ou en creux, les trois éléments ci-dessus, de les développer et d'identifier quelques-unes des raisons pour lesquelles les experts-évaluateurs construisent leurs propres indicateurs associés aux critères de variété et de complexité des situations de travail :

1. Les experts-évaluateurs mettent systématiquement leur propre expérience, leur jugement et leur raisonnement professionnel à la base du travail d'évaluation de la compétence des candidats. Nous l'avons constaté à plusieurs reprises, mais nous n'allons pas nous attarder sur cet aspect, déjà exploré par d'autres travaux.

2. Les experts-évaluateurs demandent aux responsables du processus d'évaluation en RAM de leur mettre à disposition une grille d'évaluation. Cette demande relève d'une interprétation qu'ils font de la mission évaluative qui leur est confiée. C'est également une forme de protection face au travail fourni, que de partager avec l'outil d'évaluation, dont ils ne sont pas les auteurs, la responsabilité de la réussite ou de l'échec d'un candidat, responsabilité qui est possiblement trop lourde à porter seul. 


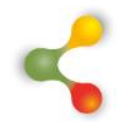

\section{REVUE HYBRIDE DE L'ÉDUCATION}

3. En tant qu'enseignants, les experts-évaluateurs sont habitués à avoir des critères d'évaluation clairs avec des indicateurs précis, qu'il leur suffit d'appliquer pour poser un verdict. C'est, selon eux, une question d'équité de l'évaluation.

4. Les experts-évaluateurs associent l'absence de rigidité de l'outil d'évaluation qui leur est mis à disposition à un manque de clarté. En réalité, l'outil prend en considération le fait que chaque dossier de RAM est unique et qu'une grille d'évaluation trop stricte ne permettrait pas de traiter de manière équitable la multitude des formes d'expression de la compétence qu'une rédaction écrite permet de mettre en valeur. II a donc été conçu sous une forme ouverte, permettant aisément des adaptations. Or, l'appropriation d'un tel outil demande un important travail préalable de conceptualisation, de compréhension des valeurs que les responsables du processus souhaitent voir développées chez les experts.

5. Les experts-évaluateurs ne disposent ni des connaissances ni du temps nécessaires au travail conceptuel préalable à la construction d'indicateurs opérationnels en partant de l'outil d'évaluation fourni. Les multiples occupations des experts-évaluateurs RAM ne leur permettent pas le luxe de cet investissement, surtout que le degré d'ouverture du dispositif laisse la possibilité de contourner l'étape de conceptualisation et construire rapidement ses propres indicateurs de compétence; sans exception, les experts-évaluateurs se saisissent de cette opportunité.

6. Les experts-évaluateurs agissent selon une éthique-en-acte qui leur est propre et qui les amène à considérer la véracité des expériences. Mayen et Tourmen (2009) montrent que les experts des jurys VAE ont souvent des doutes concernant certains acquis d'expérience des candidats à la lecture de leur dossier et que, lorsqu'ils ont la possibilité, ils vont poser aux candidats des questions spécifiques lors de l'étape suivante l'entretien. Or, le processus de RAM au BEP qui a fait l'objet de cette recherche ne prévoit pas d'entretien. L'évaluation de la compétence se fait exclusivement par une analyse de dossier. Cela montre les défis du travail d'analyse de l'expérience décrite et de traduction de l'expérience en degré de compétence. C'est une raison de plus pour prendre en compte l'activité des experts-évaluateurs expérimentés pour concevoir la formation des experts-évaluateurs novices.

\section{En termes de formation...}

En parlant de leur travail d'évaluation pour la RAM, les expertsévaluateurs ne sont pas complètement satisfaits du mode de fonctionnement qu'ils ont eux-mêmes instauré en contournant en partie l'outil d'évaluation. Ils expriment leur frustration d'avoir souvent la certitude qu'un candidat possède la compétence de travail requise, mais ne pas 


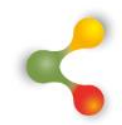

\section{REVUE HYBRIDE DE L'ÉDUCATION}

pouvoir « cocher les cases » leur permettant de valider cette compétence à l'aide de leurs propres indicateurs. Dans ce contexte, la formation et l'accompagnement pourraient faciliter l'appropriation en profondeur de la démarche d'évaluation RAM et des valeurs que les responsables du processus souhaitent retrouver chez les experts-évaluateurs. Cette formation devrait permettre aux experts de s'ouvrir à de nouvelles manières de lire, de saisir, d'analyser et d'interpréter l'expérience de travail décrite par les candidats et, à travers cette expérience, estimer la compétence qui en découle à l'aide d'indicateurs adaptés.

Toutefois, malgré le fait que la tâche d'évaluation en RAM est soumise à une subjectivité inhérente au jugement et à l'expérience des ceux qui la réalise, l'expérience de travail et l'expérience d'évaluation font des experts-évaluateurs expérimentés des référents incontournables pour la révision des critères d'évaluation. De ce point de vue, les indicateurs qui sont ressortis de cette recherche comme étant pertinents pour les expertsévaluateurs (qu'ils soient présents ou absents de l'outil d'évaluation mis à disposition) sont à étudier avec le plus grand soin afin de considérer leur place dans un nouvel outil d'évaluation et dans la formation des nouveaux experts-évaluateurs.

Enfin, cette recherche ouvre la voie à de nouvelles pistes, qu'elle n'a pas eu pour objet d'explorer : que pensent les experts-évaluateurs de l'outil d'évaluation mis à disposition? Tentent-ils de le comprendre et de se l'approprier avant de construire leur propre outil d'évaluation? Autrement dit, de quelle manière préfèrent-ils construire l'intelligibilité essentielle à l'évaluation des dossiers RAM pour des candidats qu'ils ne connaissent pas? 


\section{REVUE HYBRIDE DE L'ÉDUCATION}

\section{Références}

Allal, L. et Lafortune, L. (dir.) (2008). Jugement professionnel en évaluation: pratiques enseignantes au Québec et à Genève. Presses de l'Université du Québec.

Aubé, L., Balleux, A., Boudreault, H., Bouffard, J., Coulombe, S. Gagnon, R., Gauthier, D. Lacroix, R., Roussel, R. et Tardif, M. (2009). Reconnaître les acquis disciplinaires dans les programmes de formation à l'enseignement en formation professionnelle dans les universités québécoises. Ministère de l'Éducation, du Loisir et du Sport.

Balleux, A. (2003). La formation professionnelle : une formation pratique marquée par le passage du métier à celui de l'enseignant. Dans $A$. Balleux (dir.), La formation à l'enseignement professionnel : identité, enjeux et perspectives (p. 18-28). Actes du colloque du $20^{\circ}$ Congrès de l'Association internationale de pédagogie universitaire (AIPU). Sherbrooke, QC, Canada.

Blais, M. et Martineau, S. (2006). L'analyse inductive générale : description d'une démarche visant à donner du sens à des données brutes. Recherches qualitatives, 26(2), 1-18. http://www.recherchequalitative.qc.ca/documents/files/revue/edition reguliere/numero26 \%282\%29/blais et martineau final2.pdf

Chaumont, M. et Leroux, J. L. (2018). Le jugement évaluatif : subjectivité, biais cognitifs et postures du professeur. Réflexion pédagogique, 31(3), http://aqpc.qc.ca/sites/default/files/revue/chaumontleroux-vol.31$\underline{\text { 3.pdf }}$

Clauzard, P. (2008). Didactique professionnelle et activité enseignante. www.philippeclauzard.com/Didactiqueprofessionnelleactiviteenseignante.pdf

Coiduras, J. L. et Carrera, X. (2011). La construction du sens autour de la notion de compétence dans des dispositifs universitaires en alternance. Éducation et francophonie, 38(2), 96-112. https://www.erudit.org/fr/revues/ef/2010-v38-n2ef1516783/1002166ar.pdf

Cortessis, S. (2010). La construction d'un jugement argumenté en VAE. Congrès de l'Actualité de la recherche en éducation et formation (AREF), 1-9. Actes du colloque, Université de Genève.

Coulet, J.-C. (2011). La notion de compétence : un modèle pour décrire, évaluer et développer les compétences. Le travail humain, 74(1), 1- 


\section{REVUE HYBRIDE DE L'ÉDUCATION}

30. https://www.cairn.info/revue-le-travail-humain-2011-1-page1.htm\#

Cuvillier, B. et Faudière, L. (2009). Rencontre avec le jury de VAE : vécu et ressenti des candidats du Conservatoire national des arts et métiers. Dans P. Mayen et A. Savoyant (dir.), Élaboration et réduction de l'expérience dans la validation des acquis de l'expérience, Relief 28.

Hébrard, P. (2013). Quelle «approche par les compétences» et quels référentiels pour la formation professionnelle aux métiers de la relation humaine ?. Le travail en évolution, 30, 17-34. https://dse.revues.org/189

Holgado, O. (2015). Apports potentiels de la didactique professionnelle à la formation à l'enseignement des métiers. https://www.usherbrooke.ca/pedtice/fileadmin/sites/pedtice/docume nts/Webinaire 15-04-09.pdf

Holgado, O. (2017). Portfolio de reconnaissance des acquis de métier en formation à l'enseignement professionnel au Québec. Le regard évaluatif des experts de métier. Communication présentée au $29^{\circ}$ colloque international de l'ADMEE, Dijon, France.

Jean, A. et Étienne, R. (2013). Des gestes de résistance à une conception dramaturgique du développement professionnel fondée sur l'articulation des dipositifs? Dans M., Altet, J., Desjardins, R., Étienne, Paquay, L. et Perrrenoud, P. (dir.), Former des enseignants réflexifs : Obstacles et résistances (p. 191-212). De Bock.

Jonnaert, P. (2011). Sur quels objets évaluer des compétences? Education \& Formation, (e-296), p.31-43.

Le Boterf, G. (2000). Construire les compétences individuelles et collectives ( $7^{\mathrm{e}}$ édition). Eyrolles.

Le Boterf, G. (2017). Agir en professionnel compétent et éthique. Halte au «tout compétences»! Éthique publique, 19(1). http://journals.openedition.org/ethiquepublique/2934

Legendre, M.-F. (2001). Sens et portée de la notion de compétence dans un programme de formation. Revue de l'AQEFLS, 23(1), 12-30. http://aqefls.org/ressources/Article Legendre.PDF

Leplat, J. (1995). À propos des compétences incorporées. Éducation permanente, 2(123), 101-113. 


\section{REVUE HYBRIDE DE L'ÉDUCATION}

Mayen, P. (2009). Expérience et formation des adultes. Dans J.-M. Barbier, É. Bourgeois, G. Chapelle et J.C. Ruano-Borbalan (dir.), Encyclopédie de la formation (p. 763-780). Presses universitaires de France.

Mayen, P. et Métral, J.-F. (2008). Compétence et validation des acquis de l'expérience. Formation emploi, 101, 183-197. http://www.oce.uqam.ca/wpcontent/uploads/2015/01/1303 metral mayen competences vae.p $\underline{\mathrm{df}}$

Mayen, P., Métral, J.-F. et Tourmen, C. (2010). Les situations de travail. Référence pour les référentiels. Recherche et formation, (64), 31-46. https://journals.openedition.org/rechercheformation/191

Mayen, P. et Tourmen, C. (2009). Les jurys de VAE ont-ils quelque chose à apprendre ? Dans P. Mayen et A. Savoyant (dir.), Élaboration et réduction de l'expérience dans la validation des acquis de l'expérience, Relief 28.

Mayeux et Mayen (2009). Regards sur l'activité de jurys VAE : des prescriptions du dispositif aux pratiques en séance. Dans $P$. Mayen et A. Savoyant (dir.), Élaboration et réduction de l'expérience dans la validation des acquis de l'expérience, Relief 28.

Ministère de l'Éducation (2003). Politique d'évaluation des apprentissages : version abrégée. Gouvernement du Québec. http://www.education.gouv.qc.ca/fileadmin/site web/documents/dps e/evaluation/13-4602-03.pdf

Romainville, M. (2011). Objectivité versus subjectivité dans l'évaluation des acquis des étudiants. Revue internationale de pédagogie de l'enseignement supérieur, $27(2)$, 1-9. http://ripes.revues.org/499\#tocto1n1

Savoie-Zajc, L. (2013). Le jugement professionnel. Dans B. Gauthier (dir.), Recherche sociale de la problématique à la collecte des données (p. 337-360). Presses de l'Université du Québec.

Savoyant, A. et Mayen, P. (2009). Introduction. Dans P. Mayen et A. Savoyant (dir.), Élaboration et réduction de l'expérience dans la validation des acquis de l'expérience, Relief 28.

Vergnaud G. (1990). La théorie des champs conceptuels. Recherches en didactique des mathématiques, 10(2-3), p. 133-170.

Vergnaud, G. (2001). Forme opératoire et forme prédicative de la connaissance. Dans J. Portugais (dir.), La Notion de compétence en 


\section{$\&$}

\section{REVUE HYBRIDE DE L’ÉDUCATION}

enseignement des mathématiques, analyse didactique des effets de son introduction sur les pratiques et sur la formation. Actes de colloque, Montréal, mai 2001.

Werquin, P. (2010). Reconnaître l'apprentissage non formel et informel : résultats, politiques et pratiques. OCDE. https://www.cicic.ca/docs/oecd/rnfil.fr.pdf

Zourhlal, A. et Coulombe, S. (2014) À propos de la contribution de la reconnaissance des acquis disciplinaires aux perspectives professionnalisantes du programme de formation à l'enseignement professionnel. Dans M. Tardif et F. Desbiens (dir.), La vogue des compétences dans la formation des enseignants : bilan critique et perspective d'avenir (p. 167-183). Presses de l'Université Laval. 